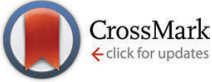

Cite this: Phys. Chem. Chem. Phys., 2016, 18, 10049

Received 23rd February 2016 Accepted 16th March 2016

DOI: $10.1039 / c 6 c p 01229 a$

www.rsc.org/pccp

\title{
Intraparticulate speciation analysis of soft nanoparticulate metal complexes. The impact of electric condensation on the binding of $\mathrm{Cd}^{2+} / \mathrm{Pb}^{2+} / \mathrm{Cu}^{2+}$ by humic acids
}

\begin{abstract}
Raewyn M. Town*a and Herman P. van Leeuwen ${ }^{\mathrm{b}}$
In aqueous dispersions of soft, charged nanoparticles, the physicochemical conditions prevailing within the particle body generally differ substantially from those in the bulk medium. Accordingly it is necessary to define intrinsic descriptors that appropriately reflect the chemical speciation inside the particle's microenvironment. Herein the speciation of divalent metal ions within the body of negatively charged soft nanoparticulate complexants is elaborated for the example case of humic acid association with $\mathrm{Cd}(॥), \mathrm{Pb}(॥)$ and $\mathrm{Cu}(I)$. The electrostatic effects are described by a two-state model that accounts for counterion condensation in the intraparticulate double layer shell at the particle/medium interface and Donnan partitioning within the bulk of the particle body. Inner-sphere complex formation is defined by an intrinsic binding constant expressed in terms of local reactant concentrations as controlled by the pertinent electrostatic conditions. For the high particle charge density case (Debye length smaller than charged site separation), three distinct intraparticulate metal species are identified, namely free hydrated ions, electrostatically condensed ions, and inner-sphere metal-humic complexes. For all metal ions studied, the electrostatic contribution to the association of the metal ion with the oppositely charged particle is found to account for a substantial fraction of the total metal bound.
\end{abstract}

\section{Introduction}

Soft and permeable nanoparticles (NPs) are ubiquitous in environmental and biological systems where they play an important role in buffering concentrations of essential and toxic ions. A basic characteristic of an aqueous dispersion of such NPs is that the reactive sites are spatially confined to the particle body which is at an electric potential different from that in the bulk medium. Accordingly, the conditions prevailing within soft charged NPs, e.g. electric potential and ion concentrations, can differ strongly from those in the bulk medium. Furthermore, the rates of their reactions with oppositely charged ionic reactants can be orders of magnitude greater than those of the corresponding molecular counterparts. ${ }^{1,2}$ This property arises from the influence of the electrostatic field of the nanoparticle on (i) the local concentrations of ionic reactant species within the NP body, and (ii) the conductive acceleration of the diffusive rate of supply of ions with opposite charge sign. The magnitude of the nanoparticulate electric field also affects equilibrium binding properties, including the extent to

\footnotetext{
${ }^{a}$ Department of Physics, Chemistry and Pharmacy, University of Southern Denmark, Campusvej 55, 5230 Odense, Denmark.E-mail: raewyn.town@sdu.dk

${ }^{b}$ Laboratory of Physical Chemistry and Colloid Science, Wageningen University,

Dreijenplein 6, 6703 HB Wageningen, The Netherlands
}

which oppositely charged ions electrostatically associate with the NP entity as well as the distribution of the metal ion over its various species and over location within the particle body, i.e. their intraparticulate speciation. Equilibrium techniques for chemical speciation analysis, such as ion-selective electrode potentiometry, generally give a measure of the concentration of free $\mathrm{M}_{\mathrm{aq}}^{2+}$ in the bulk medium; the remainder of the metal in the system is inferred to be associated with the complexant, i.e. "bound". Conventional descriptors of metal complex formation, e.g. apparent stability constants $\bar{K}_{\text {app }}$, are defined in terms of concentrations of chemical species that are averaged over the entire volume of the solution/dispersion, i.e. their smeared-out values. Evidently such parameters have limited meaning in the case of nanoparticulate complexants, where 'bound' metal stands for all physicochemical forms of $\mathrm{M}$ that are associated with the particle.

Here we develop theoretical concepts to quantify intraparticulate speciation within charged soft nanoparticulate metal ion complexants and apply these to the binding of different types of divalent metal ions by the natural heterogeneous complexant, humic acid (HA). An aqueous HA "solution" actually is a dispersion of its natural NP entities with electrokinetic features that correspond to those of highly negatively charged NPs with the complexing sites distributed over the volume of the soft and 
permeable particle body. ${ }^{3}$ At a given metal/binding site ratio, the extent to which HA binds divalent cations, and the apparent stability of the pertaining complexes, $\bar{K}_{\text {app }}$, are generally dependent on the $\mathrm{pH}$ and ionic strength of the solution medium, which points to the involvement of both electrostatic and covalent contributions to the binding. ${ }^{4}$ The extent to which divalent cations associate with $\mathrm{HA}$, as based on smeared-out equilibrium concentrations, generally follows the order $\mathrm{Ca}^{2+}<\mathrm{Cd}^{2+}<\mathrm{Pb}^{2+} \approx \mathrm{Cu}^{2+} \cdot{ }^{4-6}$ Thus, on top of the electrostatic modes of association, there is an additional contribution to the binding for $\mathrm{Cd}$ (II), $\mathrm{Pb}$ (II) and $\mathrm{Cu}$ (II) due to the formation of covalent inner-sphere complexes. At the level of $\bar{K}_{\text {app }}$ values, the various equilibrium models for metal ion speciation, e.g. the NICA-Donnan ${ }^{7}$ and $\mathrm{WHAM}^{8}$ models, incorporate both electrostatic and covalent binding parameters for the $\mathrm{M}^{2+}$-HA interaction. Typically the electrostatic effects are modeled by assuming Donnan-type partitioning of any $\mathbf{M}^{z+}$ into the HA body, ${ }^{8-14}$ with the relevant physicochemical parameters being the effective Donnan volume and the charge density of the HA particle.

Recently, we have shown that Donnan-partitioning by itself is not sufficient to explain the extent to which $\mathrm{Ca}^{2+}$ associates with HA. ${ }^{15}$ For HA nanoparticles with a high structural charge density, $\rho_{\mathrm{p}}$, and a radius $r_{\mathrm{p}}$ well above the Debye screening length, $\kappa^{-1}$, the bulk of the particle has zero net charge whilst the intraparticulate DL zone carries a strongly negative net charge in proportion to $\rho_{\mathrm{p}} \cdot{ }^{2}$ For the case of $\mathrm{Ca}^{2+}$, the net charge density in the intraparticulate part of the DL was shown to give rise to counterion condensation, analogous to that analysed by Manning for linear polyelectrolytes. ${ }^{16}$ The extent to which $\mathrm{Ca}^{2+}$ associates with a range of HA samples with different particle sizes at various ionic strengths, was well described by a simple two-state model that combines (i) purely electrostatic counterion condensation (CC) in the intraparticulate double layer (DL) at the particle/medium interface, and (ii) classical Donnan partitioning in the bulk of the HA particle. ${ }^{15}$ For the case with a 1-1 background electrolyte, the outcome is that divalent counterions may accumulate to quite high local concentrations in the DL zone. These findings are corroborated by e.g. the counterion condensation characteristics of polyelectrolytes such as DNA, ${ }^{17}$ and soft nanoparticles such as dendrimers, ${ }^{18-20}$ as well as the observation of strong counterion condensation in core-shell nanoparticles with a $3 \mathrm{D}$ structural charge in the shell. ${ }^{21}$

Since the associative reactions of $\mathrm{Ca}^{2+}$ with negatively charged ligands are dominated by electrostatics, ${ }^{22,23}$ the binding features of $\mathrm{Ca}^{2+}$ may be adopted as an approximate basis for discriminating between electrostatic and covalent contributions to binding of transition metal ions such as $\mathrm{Cd}^{2+}, \mathrm{Pb}^{2+}$, and $\mathrm{Cu}^{2+}$. The intraparticulate speciation then is more differentiated by the additional presence of inner-sphere complexes. In such cases, the application of a calcium salt as the background electrolyte is a useful means to eliminate the majority of the electrostatic contribution to the binding of the target $\mathrm{M}^{2+}$. In this paper we shall attempt to unravel the intraparticulate equilibrium metal speciation in terms of free hydrated ions, electrostatically condensed ions, and inner-sphere complexes, together with their spatial distribution within the particle body, for the case of a highly charged nanoparticulate complexant. The ensuing mode of interpretation will be applied to the humic acid complexes of $\mathrm{Cd}(\mathrm{II}), \mathrm{Pb}(\mathrm{II})$, and $\mathrm{Cu}$ (II). The type of HA selected features particles large enough to satisfy $\kappa r_{\mathrm{p}}>1$ for the ionic strengths investigated. This facilitates straightforward application of the two-state approach to particles with a Donnan type bulk core and a relatively thin double layer zone at the particle/medium interface.

\section{Theory}

\subsection{Smeared-out vs. in situ stability constants}

Conventional apparent stability constants, $\bar{K}_{\text {app }}$, are expressed in terms of smeared-out concentrations and collectively include all factors that contribute to the overall stability of the association between a metal ion and a reactive site, S, i.e. both electrostatic and covalent chemical interactions. Since HA is a chemically heterogeneous complexant, ${ }^{4}$ the equilibrium relationship between the metal ion concentration and the various binding sites is described by a distributed affinity with the apparent stability constant $\bar{K}_{\text {app }}$ depending on the degree of occupation, $\theta_{\mathbf{M}}$, of the binding sites by $\mathrm{M}^{4}{ }^{4}$ Therefore, in comparing stabilities of metal-HA complexes it is usual practice to do so at a given $\theta_{\mathbf{M}}$ value. Conventionally, $\theta_{\mathbf{M}}$ is defined as the ratio of the concentrations of 'bound metal' and available reactive sites, where 'bound metal' denotes the total concentration of the metal ions that are somehow associated with the particle, via either electrostatic and/or covalent interactions where applicable. Analysis of the intraparticulate metal speciation enables $\theta_{\mathrm{M}}$ to be defined in a more meaningful way as the ratio between the concentrations of inner-sphere metal complexes, MS, and reactive sites, i.e.

$$
\theta_{\mathrm{M}}=c_{\mathrm{MS}} / c_{\mathrm{S}, \mathrm{t}}=c_{\mathrm{MS}}^{*} / c_{\mathrm{S}, \mathrm{t}}^{*}
$$

where $c_{\mathrm{MS}}$ and $c_{\mathrm{S}, \mathrm{t}}$ are the local intraparticulate concentrations of the inner-sphere complexes and total reactive sites, respectively, and $c_{\mathrm{MS}}^{*}$ and $c_{\mathrm{S}, \mathrm{t}}^{*}$ are the smeared-out counterparts. Under conditions of low coverage of binding sites by $\mathrm{M}^{2+}$, the total reactive site concentration is approximately equal to the concentration of free reactive sites, $c_{\mathrm{S}}$.

For simple molecular ligands, the conventional reported metal complex stability constants inherently include both electrostatic and covalent contributions to the binding. The overall metal ion complexation reaction often follows the Eigen mechanism, which involves formation of an outer-sphere ion pair $\mathbf{M}_{\mathrm{aq}}^{2+} \bullet \mathrm{S}$, between the metal ion $\mathbf{M}_{\mathrm{aq}}^{2+}$ with an intact primary hydration shell and the reactive site $\mathrm{S}$, followed by dehydration and formation of an inner-sphere complex MS. ${ }^{24}$ For simple ligands, the magnitude of the electrostatic attraction between a negatively charged reactive site and a positively charged metal ion is described by the outer-sphere stability constant, $K^{\text {os }}$, which is straightforwardly computed on the basis of FuossBoltzmann electrostatics: ${ }^{25,26}$

$$
K^{\mathrm{os}}=\frac{4}{3} \pi N_{\mathrm{Av}} a^{3} \exp \left(-U^{\mathrm{os}} / k T\right)
$$

where $a$ is the center-to-center distance between $\mathbf{M}$ and $\mathrm{S}$, and $U^{\text {os }}$ is the interionic potential for an individual ion pair, given by: 


$$
U^{\mathrm{os}}=\frac{z_{\mathrm{M}} z_{\mathrm{S}} e^{2}}{4 \pi \varepsilon_{0} \varepsilon a}\left(1-\frac{\kappa a}{1+\kappa a}\right)
$$

where $z_{\mathrm{M}}$ and $z_{\mathrm{S}}$ are the charge on the metal ion and the binding site, respectively, $\varepsilon_{0} \varepsilon$ is the permittivity of the electrolyte solution, and the term $\kappa a /(1+\kappa a)$ accounts for the extent of screening involved. For a $2+/ 1-$ ion pair, $K^{\mathrm{os}}$ is of the order $10^{-3} \mathrm{~m}^{3} \mathrm{~mol}^{-1}$, which is comparable to the $K_{\text {app }}$ for the essentially electrostatic $\mathrm{Ca}^{2+}$ associate with acetate. ${ }^{27}$

The intrinsic stability constant, $K_{\text {int }}$, represents the inherent chemical affinity between a metal ion and a reactive site, after correction for the long-range electrostatics beyond those on the scale of atom-atom interactions. The intrinsic stability constant format is not often used for complexes with simple ligands, but for our present purposes of comparison with the high charge density NP complexant case, we may define $K_{\text {int }}$ as:

$$
K_{\text {int }}=K / f^{\text {os }}
$$

where $K$ is the conventional stability constant for the corresponding simple ligand, and $f^{\text {os }}$ represents the extent to which the concentration of the reactant metal ions in the outer-sphere volume is enhanced relative to that in the bulk electrolyte medium, i.e.

$$
f^{\mathrm{os}}=\exp \left(-U^{\mathrm{os}} / k T\right)
$$

For a 2+/1- ion pair such as $\mathrm{Ca}^{2+}$-acetate, with a center-to-center distance of $0.6 \mathrm{~nm}$, the values of $f^{\text {os }}$ are approximately 4 and 7 for ionic strengths of 100 and $10 \mathrm{~mol} \mathrm{~m}^{-3}$, respectively.

Intrinsic stability constants have been used to describe metal ion complexation by soft charged nanoparticulate complexants, e.g. carboxymethyldextran $^{28}$ and humic acids. ${ }^{7,8,29}$ For such complexants, the local physicochemical conditions prevailing within the particle body $(\mathrm{pH}$, reactive site concentration, electrolyte ion concentrations and effective charge screening) can be very different from those in the surrounding aqueous medium. Eigentype complex formation mechanisms have been elaborated for the nanoparticulate case, with rate constants for outer-sphere and inner-sphere complexation defined in terms of the conditions within the particle body. ${ }^{30}$ Accordingly, for soft charged NPs, it is more meaningful to define the intrinsic stability constant in terms of intraparticulate (in situ) conditions and reactant concentrations. This necessitates a detailed account for the electrostatic binding effects, as detailed below.

\subsection{Electrostatic features of HA}

Unless otherwise stated, we shall consider the case where a highly charged soft NP is equilibrated in an aqueous noncomplexing background electrolyte, with a concentration well in excess over the smeared-out concentration of charged sites. In turn, the latter is in large excess over the target divalent cation, $\mathrm{M}^{2+}$, i.e. we consider the case of low coverage of binding sites by $\mathrm{M}^{2+}$.

2.2.1 Donnan partitioning. For soft charged particles with radius much greater than the Debye screening length a Donnan potential difference, $\psi_{\mathrm{D}}$, is established between the bulk of the soft phase and the bulk aqueous medium according to: ${ }^{31}$

$$
\psi_{\mathrm{D}}=\frac{R T}{z F} a \sinh \left(\frac{\rho_{\mathrm{p}}}{2 z F c_{1}}\right)
$$

where $z_{-}=z_{+}=z$ is the valence of the symmetrical excess background electrolyte with bulk concentration $c_{1}, \rho_{\mathrm{p}}$ is the volume charge density due to charged groups on the backbone of the soft body, and other constants have their usual meaning. For the case of a 2-1 excess background electrolyte, with concentration $c_{2}, \psi_{\mathrm{D}}$ may be obtained via: ${ }^{31}$

$$
-\frac{1}{3} \exp ^{-2 F \psi_{\mathrm{D}} / R T}+\frac{1}{3} \exp ^{F \psi_{\mathrm{D}} / R T}-\frac{\rho_{\mathrm{p}}}{6 c_{2}}=0
$$

A Donnan phase necessarily requires a charge density so high that the average structural charge separation, $\ell_{\mathrm{C}}$, is small enough to satisfy the condition $\kappa \ell_{\mathrm{C}} \ll 1$ ("high charge density" regime). The magnitude of $\psi_{\mathrm{D}}$ influences the equilibrium partitioning of any type of ion $\mathrm{i}$, with valency $z_{\mathrm{i}}$, between the soft particle phase (with concentration $c_{\mathrm{i}, \mathrm{D}}$ ) and the electrolyte solution (with concentration $c_{i}^{*}$ ) according to a Boltzmann factor, $\bar{f}_{\mathrm{B}}:{ }^{32}$

$$
\bar{f}_{\mathrm{B}, \mathrm{i}}=\frac{c_{\mathrm{i}, \mathrm{D}}}{c_{\mathrm{i}}^{*}}=\exp \left(\frac{-z_{\mathrm{i}} F \psi_{\mathrm{D}}}{R T}\right)
$$

For example, for a $10 \mathrm{~mol} \mathrm{~m} \mathrm{~m}^{-3}$ 1-1 electrolyte solution a $\rho_{\mathrm{p}}$ of $-700 \mathrm{~mol} e \mathrm{~m}^{-3}$ yields a $\psi_{\mathrm{D}}$ of $-107 \mathrm{mV}$ (eqn (6)), whilst for a 2-1 electrolyte at the same ionic strength the $\psi_{\mathrm{D}}$ is $-59 \mathrm{mV}$ (eqn (7)). Accordingly, in the 1-1 electrolyte the $\bar{f}_{\mathrm{B}}$ for $1+$ ions is 70 whilst that for $2+$ ions is ca. $5 \times 10^{3}$, whereas in the $2-1$ electrolyte the $\bar{f}_{\mathrm{B}}$ values are 10 and 105 , respectively (eqn (7)). In the present work, the electrolyte concentration (at ionic strengths of either 10 or $100 \mathrm{~mol} \mathrm{~m}^{-3}$ ) is in great excess over that of the target $\mathrm{M}^{2+}$ ions (on the order of $10^{-3} \mathrm{~mol} \mathrm{~m}^{-3}$ ). Thus, in e.g. $10 \mathrm{~mol} \mathrm{~m}^{-3} 1-1$ electrolyte the local concentration of $1+$ ions in the Donnan bulk is $700 \mathrm{~mol} \mathrm{~m}^{-3}$ whilst that of the $2+$ ions is ca. $0.5 \mathrm{~mol} \mathrm{~m}^{-3}$. In spite of its much larger $\bar{f}_{\mathrm{B}}$, the local concentration of $\mathrm{M}^{2+}$ in this example is still insignificant compared to the $1+$ ions in their neutralizing practically all of the structural charge of the particle body.

2.2.2 Ion pairing. An ion pair comprises the purely electrostatic associate between a cation and a negatively charged site. In the first step of Eigen type aqueous complex formation reactions the primary hydration shell of the cation remains intact and the pertaining ion-site pair intermediate is usually denoted as "outer-sphere complex". ${ }^{24}$ Outer-sphere ion pairing may occur throughout the particle body but this is of an essentially non-electrostatic nature for particles in the so-called "high charge density" regime. The intraparticulate electric potential profile then approaches an approximately constant value (see Fig. 1), the characteristic of a true Donnan phase. ${ }^{2}$ As a consequence, the outer-sphere volume around a complexing site and the remaining aqueous volume of the particle are at practically the same potential. In such a case the intraparticulate free metal ion and the outer-sphere associates are indistinguishable, i.e. they are electrostatically nearly equivalent and interconversion 


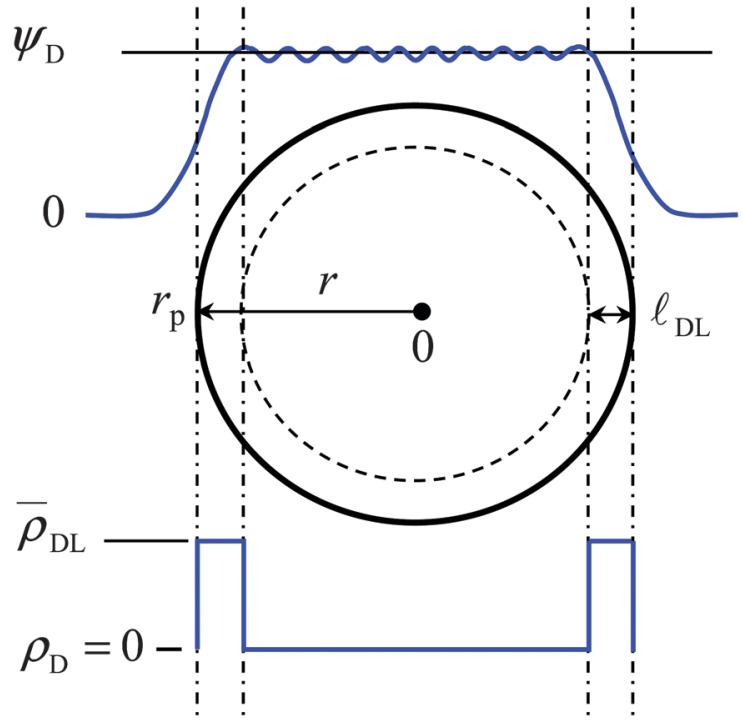

Fig. 1 Schematic representation of the two-state electrostatic model for a soft spherical nanoparticle with radius $r_{\mathrm{p}}$ and condensation layer thickness $\ell_{\mathrm{DL}}$, for the high charge density case $\left(r_{\mathrm{p}}>\kappa_{\mathrm{p}}^{-1}>\ell_{\mathrm{C}}\right)$. The model parameter $\ell_{\mathrm{DL}}$ is related to the intraparticulate $\kappa_{\mathrm{p}}^{-1}$. The true profile of the potential, $\psi$, is shown in blue above the NP, with $\psi_{D}$ corresponding to the average of the rippled profile; $\psi=0$ for the bulk medium (dilute dispersion of HA). The intraparticulate profile of the charge density, $\rho$, is sketched in blue below the NP; the profile of $\rho$ in the extraparticulate double layer in the medium is of a classical diffuse nature. In the case of negatively charged NPs, both $\psi_{\mathrm{D}}$ and $\bar{\rho}_{\mathrm{DL}}$ have a negative sign.

between these species is very fast. The probability for an intraparticulate cation to be within the outer-sphere zone is then simply determined by the volume ratio, $n_{\mathrm{S}} V^{\mathrm{os}} / V_{\mathrm{p}}$, where $n_{\mathrm{S}}$ is the number of sites in the particle body, $V^{\text {os }}$ the outer-sphere volume around a site and $V_{\mathrm{p}}$ is the volume of the particle.

2.2.3 Counterion condensation. Electrostatic condensation theory, as originally developed by Manning for linear polyelectrolytes, ${ }^{16}$ describes the tendency of the polyion to condense counterions in its immediate vicinity if the structural charge density is beyond certain limiting values. Condensed ions remain fully or partly hydrated and are mobile within a limited spatial zone of condensation. As detailed previously, ${ }^{15}$ Manning's expressions for the 1D case should not be expected to quantitatively apply to HA's 3D arrangement of structural charge. Indeed, a charge separation of $c a .2 \mathrm{~nm}$ in the $3 \mathrm{D}$ particle body translates to an effective 1D charge separation of ca. $0.5 \mathrm{~nm}$, highlighting that condensation in the 3D site distribution structure occurs at charge separations greater than for the $1 \mathrm{D}$ case. ${ }^{15}$ On the other hand, the DL zone of a highly charged particle will have a thickness on the order of a nm, ${ }^{33}$ implying that the charged shell at the interface should not be too far away from a $2 \mathrm{D}$ charge distribution. This makes it understandable that the inclusion of a condensation limit higher than its 1D value resulted in a fairly satisfactory Manning type description of electrostatic ion binding to soft spherical HA NPs, whilst the remaining net charge in the DL zone was in broad agreement with the potential change across the particle/ medium interface. ${ }^{15}$
2.2.4 Two-state model for electrostatic ion binding. The two-state CC-Donnan model for electrostatic ion binding by HA combines Donnan partitioning within the uncharged bulk zone of the soft NP (volume $V_{\mathrm{D}}$ ) with counterion condensation in the negatively charged intraparticulate double layer zone of thickness $\ell_{\mathrm{DL}}$ (and volume $V_{\mathrm{DL}}$ ). ${ }^{15}$ The model divides the volume of an individual particle, with radius $r_{\mathrm{p}}$ and the center of the particle at $r=0$, into two zones (Fig. 1) comprising:

(i) an intraparticulate condensation shell, with thickness $\ell_{\text {DL }}$, over which the complete intraparticulate double layer charge is evenly distributed $\left(\rho=\bar{\rho}_{\mathrm{DL}}\right)$, so that charge density driven counterion condensation may be invoked. The magnitude of $\ell_{\mathrm{DL}}$ is related to the intraparticulate Debye length, $\kappa_{\mathrm{p}}^{-1}$, for which values have been estimated by Duval. ${ }^{33}$ Within the model, the potential $\psi_{\mathrm{DL}}$ in the condensation zone has a linear profile as arising from the averaged net charge density, $\bar{\rho}_{\mathrm{DL}}$.

(ii) the remaining interior volume of the soft nanoparticle, with volume $V_{\mathrm{D}}$, carries no net charge and is considered to behave as a true Donnan phase with a constant potential, $\psi_{\mathrm{D}}$, throughout.

Accordingly, the two-state approximation is formulated by the set of conditions for the condensation volume, eqn (9), and the Donnan volume, eqn (10):

$$
\begin{gathered}
\left(r_{\mathrm{p}}-\ell_{\mathrm{DL}}\right)<r<r_{\mathrm{p}}: \rho=\bar{\rho}_{\mathrm{DL}} \\
0<r<\left(r_{\mathrm{P}}-\ell_{\mathrm{DL}}\right): \rho=0 \text { and } \psi=\psi_{\mathrm{D}}
\end{gathered}
$$

The particle volume fraction of the DL shell is denoted by $\varphi_{\mathrm{DL}}$ and that of the Donnan volume by $\varphi_{\mathrm{D}}$. Note that the assumption of a constant potential throughout the Donnan volume of the particle requires a volume fraction ratio $\varphi_{\mathrm{DL}} / \varphi_{\mathrm{D}}$ much less than unity. That is, $\kappa r_{\mathrm{p}}$ should be well above unity, or more rigorously $r_{\mathrm{p}}$ should be much larger than the intraparticulate electric double layer thickness, $\kappa_{\mathrm{p}}^{-1}$. Since $\kappa_{\mathrm{p}}^{-1}$ is typically smaller than $\kappa^{-1,33}$ this amounts to a lower minimum value for $r_{\mathrm{p}}$. In this work we focus on HA entities for which $\varphi_{\mathrm{DL}} / \varphi_{\mathrm{D}}$ is less than 0.1.

\subsection{Intraparticulate metal species}

The reactive sites and inner-sphere complexes are physically present only within the nanoparticle body: their in situ concentrations, $c_{\mathrm{S}}$ and $c_{\mathrm{MS}}$, respectively, are high, but their ratio is the same as that of the smeared-out concentrations, $c_{\mathrm{S}}^{*}$ and $c_{\mathrm{MS}}^{*}$. The free metal ion exists both in the bulk aqueous medium and within the particle body, but its equilibrium concentration will be very different in the two domains. For a nanoparticulate complexant the intrinsic stability constant of the inner-sphere complex MS, $\bar{K}_{\text {int }}$, is defined as:

$$
\bar{K}_{\text {int }}=\frac{c_{\mathrm{MS}}}{c_{\mathrm{S}} c_{\mathrm{M}, \mathrm{f}}}
$$

where $c_{\mathrm{M}, \mathrm{f}}$ and $c_{\mathrm{S}}$ are the local average concentrations of free metal ion and reactive sites in the particle body, respectively, and the bar in $\bar{K}_{\text {int }}$ signifies that, for the present case of a chemically heterogeneous complexant, the intrinsic stability represents a weighted average of all the inner-sphere complexes that are formed at the applicable $\theta_{\mathrm{M}}$. The computation of $\bar{K}_{\text {int }}$ uses the 
Table 1 Parameters defining the bulk and intraparticulate metal speciation in dispersions of highly charged, soft nanoparticulate complexants ${ }^{a}$

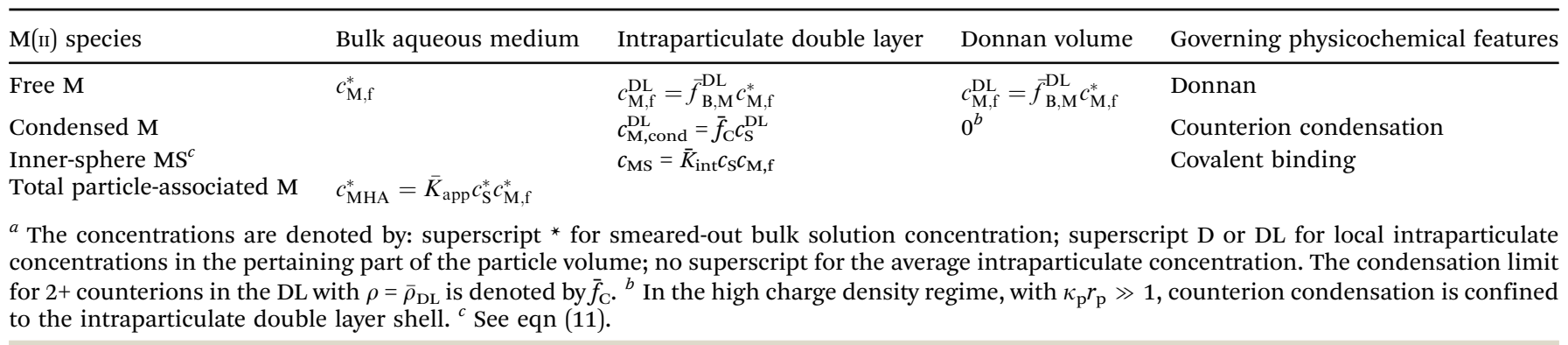

average intraparticulate concentrations of free $\mathrm{M}_{\mathrm{aq}}{ }^{2+}$ and $\mathrm{S}$, which in the high charge density regime (with $\kappa_{\mathrm{p}} r_{\mathrm{p}} \gg 1$ ) are practically equal to the concentrations in $V_{\mathrm{D}}$; in $V_{\mathrm{DL}}$, that is near the interface with the aqueous medium, the concentration of hydrophilic sites may be somewhat higher and the free metal ion concentration will be lower due to the lower $\bar{f}_{\mathrm{B}, \mathrm{M}}$. The ensuing metal ion speciation scheme for high charge density soft NP complexants is given in Table 1.

\section{Experimental}

\subsection{Properties of $\mathrm{HA}$}

The soil HA sample was from Aldrich and the particles have an approximately constant particle radius, $r_{\mathrm{p}}$, of $c a .80 \mathrm{~nm}$ over the $\mathrm{pH}$ range 5 to 8 and ionic strength range 0 to $400 \mathrm{~mol} \mathrm{~m}^{-3} \mathrm{KCl}^{34,35} \mathrm{In}$ general, experimental estimates of the particle size of various types of HA by dynamic light scattering, capillary electrophoresis, and fluorescence correlation spectroscopy do not provide any evidence for significant changes in the particle size as a function of ionic strength in the range 0 to $200 \mathrm{mM} .^{35-37}$ The chosen Aldrich HA entities conform to $\kappa_{\mathrm{p}} r_{\mathrm{p}} \gg 1$, which facilitates straightforward application of the CC-Donnan approach (see Section 2). It is considered unlikely that the present HA entities, with $r_{\mathrm{p}}$ of $80 \mathrm{~nm}$, are composed of conglomerates of smaller entities, each with its own independent DL. The point is that such a situation should feature extensive aggregation/disaggregation upon changing $\mathrm{pH}$ and ionic strength, in contrast to experimental observation. ${ }^{34}$ The total carboxyl group content of this HA is $c a .3 \mathrm{~mol}$ per $\mathrm{kg}$ of HA. ${ }^{38,39}$ The effective charge density within the particle is ca. $-700 \mathrm{~mol} e \mathrm{~m}^{-3}$ in an electrolyte medium at $\mathrm{pH} 6$, corresponding to $\mathrm{ca} .9 \times 10^{5} \mathrm{COO}^{-}$per particle. Within the particle body the $\mathrm{pH}$ will be at least 1 unit below that in the bulk medium, and thus carboxylate groups will be the major structural charge carriers and reactive sites under our experimental conditions. By estimating the outer-sphere radius to be $0.6 \mathrm{~nm},{ }^{26}$ and taking into account that the $\mathrm{COO}^{-}$is attached to the HA structure, the outer-sphere volume/ particle volume ratio $n_{\mathrm{S}} V^{\mathrm{os}} / V_{\mathrm{p}}$ comes to $c a$. 0.5 . That is, approximately half of the intraparticulate free $\mathbf{M}_{\mathrm{aq}}^{2+}$ is occupying an outer-sphere ion pair position, implying that each individual intraparticulate $\mathbf{M}_{\mathrm{aq}}^{2+}$ spends about half of its time as an ion pair with $\mathrm{COO}^{-}$.

\subsection{Reagents and solution conditions}

Solutions were buffered to pH 6 with $1 \mathrm{~mol} \mathrm{~m}^{-3}$ MES buffer (2-( $N$-morpholino)-ethanesulfonic acid) prepared from the solid (Fluka, MicroSelect, $\geq 99.5 \%$ ): MES buffer has a low affinity for
$\mathrm{Cd}(\mathrm{II}), \mathrm{Pb}(\mathrm{II})$ and $\mathrm{Cu}(\mathrm{II}) .^{40,41}$ Ionic strength was maintained at $10 \mathrm{~mol} \mathrm{~m}{ }^{-3}$ or $100 \mathrm{~mol} \mathrm{~m}{ }^{-3}$ with $\mathrm{KNO}_{3}$ or $\mathrm{Ca}\left(\mathrm{NO}_{3}\right)_{2}(\mathrm{BDH}$, AnalaR). M(II) solutions were prepared by dilution of standards: $\mathrm{Cd}$ (II) and $\mathrm{Cu}$ (II) standards were from Aldrich; $\mathrm{Pb}$ (II) standard was from Metrohm. In all experiments in this work, the concentration of $\mathrm{HA}$ in the dispersion was $c a .50 \mathrm{~g} \mathrm{~m}^{-3}$, corresponding to a volume fraction on the order of $10^{-4}$ (determined using the dry HA density of $1.66 \mathrm{~kg} \mathrm{~m}^{-3}$ and an $80 \%$ water content). The total concentration of $\mathrm{M}(\mathrm{II}), c_{\mathrm{M}, \mathrm{t}}^{*}$, was $4.6 \times 10^{-3} \mathrm{~mol} \mathrm{~m}^{-3}$ so that there is a $c a .20$ fold excess of binding sites over total metal in the dispersion. All solutions were prepared in ultrapure deionized water (resistivity $>18 \mathrm{M} \Omega$ $\mathrm{cm}$ ) from a Milli-Q gradient system.

\subsection{Electrochemical measurements}

Stripping chronopotentiometry at scanned deposition potential $(\mathrm{SSCP})^{42}$ and Absence of Gradients and Nernstian Equilibrium Stripping (AGNES) ${ }^{43}$ were used to determine the concentration of free $\mathbf{M}_{\mathrm{aq}}^{2+}$ in the bulk medium, $c_{\mathrm{M}, \mathrm{f}}^{*}$, and thus the total concentration of $\mathbf{M}$ associated with the HA particles, $c_{\mathrm{MHA}}^{*}$. Electrochemical measurements were performed with an Ecochemie $\mu$ Autolab potentiostat coupled with a Metrohm 663 VA stand. The oxidation step was performed under complete depletion conditions (stripping current $=2 \mathrm{nA}$ ). ${ }^{44}$ The electrometer input impedance of these instruments is $>100 \mathrm{G} \Omega$. The working electrode was a Metrohm multimode mercury drop electrode, the auxiliary electrode was glassy carbon, and the reference electrode was $\mathrm{Ag}|\mathrm{AgCl}| \mathrm{KCl}(\mathrm{sat})$ encased in a $100 \mathrm{~mol} \mathrm{~m}^{-3} \mathrm{KNO}_{3}$ jacket. Solutions were initially purged with oxygen-free $\mathrm{N}_{2}$, then a nitrogen blanket was maintained during measurements.

\subsection{Intraparticulate speciation analysis}

For CC-Donnan computations, the volume fraction of HA in the dispersion was used to convert smeared-out concentrations into local ones. The intraparticulate speciation (see Table 1) is computed by satisfying the electrostatic demands (counterion condensation and Donnan partitioning) and ascribing the remaining $\mathbf{M}$ to inner-sphere complexes. The electrostatic binding includes the accumulation of $\mathrm{M}^{2+}$ according to the pertaining $\bar{f}_{\mathrm{B}}$ for Donnan partitioning in $V_{\mathrm{D}}$ (eqn (8)) and Boltzmann accumulation in the $V_{\mathrm{DL}}$, and the condensation of $\mathrm{M}^{2+}$ to compensate for some prescribed fraction of the charge in the DL. For the present experimental conditions, this fraction was shown to be about $80 \%$ of the available $700 \mathrm{~mol} \mathrm{~m}^{-3}$, 
corresponding to a local $\mathrm{M}^{2+}$ concentration of $280 \mathrm{~mol} \mathrm{~m}^{-3}$ (the contribution from monovalent cations is negligible ${ }^{45}$ ); see Results and Discussion below for details on this value of the condensation fraction for $2+$ counterions. The presence of MS in the DL reduces the net charge therein, and thus the condensation demand is correspondingly decreased. Accordingly, the initial intraparticulate metal speciation is iterated with respect to the concentrations of inner-sphere complexes versus condensed metal ions in the DL zone until a consistent intraparticulate distribution over the Donnan bulk and DL zone is attained. Consistent values for $c_{\mathrm{MS}}$ and $c_{\mathrm{M} \text {,cond }}^{\mathrm{DL}}$ were attained after $c a .4$ iterations in all cases.

\section{Results and discussion}

\subsection{Electrostatic contribution to the binding}

The association of $\mathrm{Ca}^{2+}$ with $\mathrm{HA}$ is dominated by electrostatic binding. ${ }^{22,23}$ This feature provides us with a useful tool in the analysis of the HA complexes of transition metal ions that generally do involve covalent chemical bonds in addition to the electrostatic binding similar to that for $\mathrm{Ca}^{2+}$. Foremost, the analysis of data on the extent of association of $\mathrm{Ca}^{2+}$ with several HA samples under a range of $\mathrm{pH}$ and ionic strength conditions enables us to establish the magnitudes of the electrostatic parameters for the CC-Donnan model, i.e. the thickness of the DL shell, $\ell_{\mathrm{DL}}$, and the extent of condensation in the DL zone, $\bar{f}_{\mathrm{C}}$. Furthermore, the use of data for $\mathrm{Cd} / \mathrm{Pb} / \mathrm{Cu}$ with a calcium salt as the background electrolyte allows for the elimination of a great deal, if not all, of the electrostatic contribution to the binding of the target $\mathrm{M}^{2+}$ : this strategy provides a means to test the consistency of our approach for the determination of $\bar{K}_{\text {int }}$. These issues are elaborated in the following sections.

For the given experimental conditions in the present work, the HA entities carry a charge density of approximately $-7 \times$ $10^{7} \mathrm{C} \mathrm{m}^{-3}$, i.e. $0.7 \mathrm{~mol} e$ per $\mathrm{dm}^{3}$. For the chosen ionic strengths of 10 and $100 \mathrm{~mol} \mathrm{~m}^{-3}$, the HA charge density gives rise to Donnan potentials $\psi_{\mathrm{D}}$ of $c a .-100 \mathrm{mV}$ and $-50 \mathrm{mV}$, respectively in 1-1 electrolyte (eqn (6)). The ensuing Boltzmann factors for equilibrium partitioning of the free metal ion between the bulk medium and the intraparticulate zones were computed using eqn (8). For the conditions considered herein, the amount of free $\mathrm{M}_{\mathrm{aq}}^{2+}$ accumulated in the intraparticulate DL is an insignificant proportion of the total intraparticulate $\mathrm{M}$, thus for present purposes it is sufficient to estimate the extent of Boltzmann accumulation in this zone by taking the potential at the particle/ medium interface to be $c a$. $2 / 3$ of the Donnan potentials. ${ }^{46,47}$ Conductivity data have shown that divalent ions such as $\mathrm{Ca}^{2+}$ and $\mathrm{Ba}^{2+}$ exhibit condensation behavior. ${ }^{48}$ In line with that, the two-state CC-Donnan model for electrostatic ion binding was applied to ion-selective electrode measurements of $\mathrm{Ca}^{2+}$ binding by a forest soil $\mathrm{HA}$ at $\mathrm{pH} 6$ in 1-1 electrolyte at $I=10$ and $100 \mathrm{~mol} \mathrm{~m}^{-3}\left(r_{\mathrm{p}} c a .30 \mathrm{~nm}\right),{ }^{49}$ and Aldrich $\mathrm{HA}$ at $\mathrm{pH} 8$, $I=80 \mathrm{~mol} \mathrm{~m}^{-3} \cdot{ }^{50}$ An $\ell_{\mathrm{DL}}$ of $c a .2 \mathrm{~nm}$ with $20 \%$ uncompensated charge remaining in the DL, together with Boltzmann accumulation in the intraparticulate DL and Donnan partitioning in the remainder of the particle body, provided a good description of the binding data at both ionic strengths and $\mathrm{pH}$ values. More specifically, an uncompensated charge of $-140 \mathrm{~mol} e \mathrm{~m}^{-3}$ (20\% of the structural charge) within a DL thickness, $\ell_{\mathrm{DL}}$, of $2 \mathrm{~nm}$ in a particle of radius $80 \mathrm{~nm}$ corresponds to a surface charge, $\sigma_{\mathrm{s}}$ of $-0.025 \mathrm{C} \mathrm{m}^{-2}$. The corresponding potential drop over the intraparticulate DL $\left(=\sigma_{\mathrm{s}} \ell_{\mathrm{DL}} / \varepsilon \varepsilon_{0}\right)$ of $c a .-70 \mathrm{mV}$ is of the correct order of magnitude, certainly if it is taken into account that the density of deprotonated carboxyl groups is highest in the more hydrophilic outer regions of the HA particle body. ${ }^{51}$ That is, the CC-Donnan approach describes the $\mathrm{Ca}^{2+}-\mathrm{HA}$ association purely in terms of an electrostatic partition equilibrium and provides a consistent description of $\mathrm{Ca}^{2+}-\mathrm{HA}$ association for various HA samples under different conditions of $\mathrm{pH}$ and ionic strength. ${ }^{15}$ The above parameters established for $\mathrm{Ca}^{2+}$ binding were then applied as input parameters to define the electrostatic contribution to the binding of $\mathrm{Cd}^{2+}, \mathrm{Pb}^{2+}$, and $\mathrm{Cu}^{2+}$.

\subsection{Smeared-out metal complexation parameters}

Until now, metal ion binding by nanoparticulate complexants such as HA has been interpreted in terms of so-called smearedout parameters. That is, the apparent stability constant, $\bar{K}_{\text {app}}$, is defined in terms of the concentration of free metal ions in the bulk medium, together with the total smeared-out concentration of all forms of $\mathrm{M}$ that are associated with the particle, and the smeared-out reactive site concentration. At the level of the bulk solution parameters, clear differences are observed between the complexation behavior of various metal ions. E.g. for a given solution composition, the free metal ion concentration in the bulk medium follows the order $\mathrm{Cd}(\mathrm{II})>$ $\mathrm{Pb}(\mathrm{II}) \approx \mathrm{Cu}$ (II). Also, the features of the SSCP waves (characteristic potential, shape, limiting signal) for the mentioned metal ions are modified to different extents in the presence of HA, indicating differences in the stability and lability of their HA complexes. Reported $\bar{K}_{\text {app }}$ values typically follow the order $\mathrm{Cd}(\mathrm{II})<\mathrm{Pb}$ (II) $\approx \mathrm{Cu}$ (II), with values obtained at $I=10 \mathrm{~mol} \mathrm{~m}^{-3}$ being greater than those at $100 \mathrm{~mol} \mathrm{~m}^{-3} \cdot 52,53$ For Cd(II) binding with Aldrich HA at pH 6 at $I=100 \mathrm{~mol} \mathrm{~m}{ }^{-3}, \bar{K}_{\text {app }}=c a .20 \mathrm{~m}^{3} \mathrm{~mol}^{-1},{ }^{54}$ and at $I=10 \mathrm{~mol} \mathrm{~m}^{-3}, \bar{K}_{\text {app }}=c a .100 \mathrm{~m}^{3} \mathrm{~mol}^{-1}{ }^{55} \mathrm{The} \bar{K}_{\text {app }}$ values for $\mathrm{Cu}$ (II) and $\mathrm{Pb}$ (II) binding to various $\mathrm{HA}$ at $\mathrm{pH} 6$, with a $c a$. 20 -fold excess concentration of reactive sites over total metal ions, typically are in the range of $10^{3}$ to $10^{4} \mathrm{~m}^{3} \mathrm{~mol}^{-1} \cdot{ }^{38,56-58}$ It should be noted, though, that the increase in $\bar{K}_{\text {app }}$ is not due to a corresponding increase in binding strength, but rather reflects the increase in intraparticulate free $\mathrm{M}$ with decreasing ionic strength. This is illustrated in Table 2 by the greater fraction of free $\mathrm{M}$ in $V_{\mathrm{D}}$ at $I=10 \mathrm{~mol} \mathrm{~m}^{-3}$. Obviously, a more rigorous description of inner sphere M-HA complex formation requires knowledge of the speciation inside the HA particle entity.

\subsection{Intraparticulate speciation and intrinsic stability constants}

The parameters defining the speciation scheme are given in Table 1. The CC-Donnan approach distinguishes between three 
types of intraparticulate metal species, namely free hydrated ions, electrostatically condensed ions, and inner-sphere complexes. Table 2 collates the concentrations of the various metal species and the intraparticulate $\bar{K}_{\text {int }}$ values as derived from local concentrations after accounting for the electrostatic effects. The data show that at the given $\theta_{\mathrm{M}}$, the free hydrated metal ions generally are a minor proportion of the total intraparticulate $\mathrm{M}$. The results for $\mathrm{Cd}$ (II) at $I=100 \mathrm{~mol} \mathrm{~m}^{-3} \mathrm{KNO}_{3}$ show that $70 \%$ of the uncomplexed $\mathrm{M}$ is condensed in the intraparticulate double layer and $30 \%$ is inner-sphere bound, whilst $\mathrm{Pb}$ (II) and $\mathrm{Cu}$ (II) are approximately equally distributed between these two forms. However, the free $\mathrm{M}_{\mathrm{aq}}^{2+}$ concentrations differ by orders of magnitude, and so does the ensuing intrinsic stability of the inner-sphere complexes, $\bar{K}_{\text {int }}$. Notably, in contrast to the trend in $\bar{K}_{\text {app }}$, the $\bar{K}_{\text {int }}$ values are lower at lower ionic strength, even though the total intraparticulate metal concentrations are the same or greater for all metal ions. This phenomenon might be related to several differences in the intraparticulate environment, including a higher free metal ion concentration at lower ionic strength, a lower free energy difference between the free $\mathrm{M}_{\mathrm{aq}}^{2+}$ and the inner-sphere complex MS at the more negative $\psi_{\mathrm{D}}$, as well as differences in the effective charge screening.

The above observations highlight the significant role of electrostatic contributions in the binding of diverse metal ions by HA. For the purely electrostatic $\mathrm{Ca}^{2+}$ case, the condensed fraction represents the dominant intraparticulate species: ca. $90 \%$ of the particle-bound $\mathrm{Ca}^{2+}$ is condensed in the DL. ${ }^{15}$ Table 2 shows that for $\mathrm{Cd}(\mathrm{II}), \mathrm{Pb}(\mathrm{II})$, and $\mathrm{Cu}(\mathrm{II})$ in $\mathrm{KNO}_{3}$ electrolyte the amounts of ions that are condensed in the DL are still a major proportion of the total, ranging from 50 to $70 \%$. This largely explains the similarity observed in the total local concentrations for different types of metal ions with different inner sphere complexation strengths. We highlight that for the present high charge density case, there is significant profiling in the concentration of $\mathrm{M}$ within the particle: the condensed ions are located within a small proportion of the total particle volume $\left(\varphi_{\mathrm{DL}}=0.07\right)$. The fraction of condensed ions corresponds to a local concentration on the order of $250 \mathrm{~mol} \mathrm{~m}^{-3} \mathrm{M}_{\mathrm{aq}}^{2+}$,ond within the DL shell, i.e. a few \% of the DL volume. ${ }^{59}$ This observation is in line with counterion condensation characteristics of polyelectrolytes such as DNA, for which divalent counterions may reach local concentrations on the order of $300 \mathrm{~mol} \mathrm{~m}^{-3}$ within a cylindrical condensation shell of thickness $c a .2 \mathrm{~nm}^{.{ }^{17}}$

If the electrostatic contribution to metal ion complexation by $\mathrm{HA}$ is properly taken into account, then the CC-Donnan derived $\bar{K}_{\text {int }}$ values given in Table 2 will correspond to the truly intrinsic stability constant for the pertaining inner-sphere complex at the given intraparticulate conditions. As long as the ionic strength in the medium is well below the structural charge density inside the nanoparticle body, $\bar{K}_{\text {int }}$ is expected to be approximately constant for given conditions of $\theta_{\mathrm{M}}, \mathrm{pH}$ and $I$ since the effective intraparticulate ionic strength is bound to be governed by the particle's charge density. To further explore the consistency of the CC-Donnan approach in its accounting for electrostatic contributions to the binding, experiments on $\mathrm{Cd}(\mathrm{II}) / \mathrm{Pb}(\mathrm{II}) / \mathrm{Cu}$ (II) $\mathrm{HA}$ complexes were performed in $\mathrm{Ca}\left(\mathrm{NO}_{3}\right)_{2}$ 
background electrolyte at the same ionic strengths as used for the measurements in $\mathrm{KNO}_{3}$ electrolyte. The concentration of $\mathrm{Ca}^{2+}$ then is a factor of several thousand times greater than that of the target $\mathrm{M}^{2+}$. Under these conditions it may be assumed that the purely electrostatic counterion condensation requirements of the HA entity are met by $\mathrm{Ca}^{2+}$ and thus the intraparticulate speciation of $\mathrm{M}^{2+}$ would comprise only Donnan-type partitioning in $V_{\mathrm{D}}$, Boltzmann accumulation in $V_{\mathrm{DL}}$ and inner-sphere complexation in both $V_{\mathrm{D}}$ and $V_{\mathrm{DL}}$. The results given in Table 2 indeed show that the $\bar{K}_{\text {int }}$ values derived from measurements in $\mathrm{Ca}\left(\mathrm{NO}_{3}\right)_{2}$ are broadly comparable to those for measurements in $\mathrm{KNO}_{3}$. The remaining differences in $\bar{K}_{\text {int }}$ between the two media should be ascribed to side effects from the differences in the pertaining Donnan potentials, the ensuing differences in $\mathrm{pH}$, the resulting small differences in $\theta_{\mathbf{M}}$, and in the effective local charge screening. Note for example that the differences in $\theta_{\mathrm{M}}$ between the $\mathrm{KNO}_{3}$ and $\mathrm{Ca}\left(\mathrm{NO}_{3}\right)_{2}$ media are greater at the lower ionic strength due to the greater change in the electrostatic contribution to the binding (Table 2).

\section{Conclusions and outlook}

The scheme developed herein for intraparticulate speciation is a rigorous and dedicated breakdown of the data into the properties of soft nanoparticulate multi-site metal ion complexants, tailored to their size and charge density. It establishes an operational basis for differentiating between electrostatic and covalent contributions to metal ion binding. The conceptual framework divides the particle body into the negatively charged intraparticulate double layer zone (volume $V_{\mathrm{DL}}$ ) that allows for counterion condensation to an extent dictated by the particle's structural charge density, and a uncharged bulk Donnan phase (volume $V_{\mathrm{D}}$ ). Three different physicochemical forms of $\mathrm{M}$ are identified, namely (i) free $\mathrm{M}_{\mathrm{aq}}^{2+}$, distributed within $V_{\mathrm{D}}$ and $V_{\mathrm{DL}}$ according to the electrostatic potential in each spatial zone, (ii) electrostatically condensed $\mathrm{M}_{\mathrm{aq}}^{2+}$ within the DL, and (iii) innersphere MS, randomly distributed throughout the entire particle volume. The eventual intraparticulate speciation is a consequence of the interplay between the electrostatic and chemical binding options for $\mathrm{M}^{2+}$ at the given local densities of total charges and reactive sites. As long as the system is not at equilibrium, the timescale over which the electrostatic relaxation processes (both Donnan partitioning and counterion condensation) take place relative to that for the chemical reactions must be taken into account. ${ }^{60}$ Although the various ion redistribution processes may be limiting the rate of complex formation for $\mathrm{Cd}(\mathrm{II}), \mathrm{Pb}$ (II) and $\mathrm{Cu}$ (II), they do not affect the eventual equilibrium situation treated herein.

Along with the analysis of in situ metal speciation for complexant particles with high structural charge density, we present an approach for the computation of intrinsic complex stability constants, $\bar{K}_{\text {int }}$. These are essentially free from longrange electrostatic contributions to the binding, i.e. they reflect the inherent strength of inner-sphere complexes in the local intraparticulate medium. $\bar{K}_{\text {int }}$ is expressed in terms of the concentrations that prevail within the soft nanoparticle body and thus facilitates insights into fundamental features of HAs.
The distinction between $\bar{K}_{\text {int }}$ and smeared-out apparent complexation constants ${ }^{7-14}$ encompasses more than the Boltzmann correction of the free metal ion concentration and/or a volume fraction conversion. As confirmed by our comparison of experimental affinity data for transition metal ions in $\mathrm{KNO}_{3}$ and in $\mathrm{Ca}\left(\mathrm{NO}_{3}\right)_{2}$ background electrolyte, condensation of the target ion $\mathrm{M}^{2+}$ in the charged DL zone plays a major role if the HA particle charge density is high. The present analysis of the ensuing intraparticulate metal speciation might lead the way to more rigorous understanding of the chemical heterogeneity of particulate HA complexes, based on intrinsic stability constants and degrees of coverage representing the true ratios between inner-sphere bound metal and reactive site concentrations. Extension of the experimental work to a broad range of $\theta_{\mathbf{M}}$ values will be crucial in that respect.

The present study has considered the high charge density regime and particle sizes well above the Debye length. Extension of the CC-Donnan approach to lower charge densities and/or $\kappa r_{\mathrm{p}}$ around or below unity is underway and may unearth more fundamental issues pertinent for both equilibrium speciation modeling and dynamic aspects of M-HA complexes. Such developments necessarily call for a quantitative description of $3 \mathrm{D}$ condensation phenomena, as well as rigorous definition of the intraparticulate double layer thickness. Furthermore, fundamental knowledge of intraparticulate speciation is also a prerequisite for comprehensive interpretation of the chemodynamic features of M-HA systems, involving consideration of the various intraparticulate and extraparticulate diffusive and kinetic fluxes of metal species. The chemodynamics are relevant for the applicability of analytical techniques such as voltammetries including various stripping techniques, ${ }^{61}$ as well as for reactivity and bioavailability features. ${ }^{62-64}$

\section{Symbols and abbreviations}

\begin{tabular}{|c|c|}
\hline AGNES & $\begin{array}{l}\text { Absence of gradients and Nernstian equilibrium } \\
\text { stripping }\end{array}$ \\
\hline $\mathrm{CC}$ & Counterion condensation \\
\hline $\mathrm{D}$ & Donnan phase \\
\hline DL & Double layer \\
\hline HA & Humic acid \\
\hline I & Ionic strength \\
\hline$S$ & Reactive site \\
\hline NP & Nanoparticle \\
\hline SSCP & $\begin{array}{l}\text { Stripping chronopotentiometry at scanned } \\
\text { deposition potential }\end{array}$ \\
\hline $\mathrm{M}_{\mathrm{aq}}^{2+} \bullet \mathrm{S}$ & Outer-sphere ion pair \\
\hline$c_{\mathrm{M}, \mathrm{f}}^{*}$ & $\begin{array}{l}\text { Concentration of free metal ion in bulk } \\
\text { electrolyte medium }\left(\mathrm{mol} \mathrm{m}^{-3}\right)\end{array}$ \\
\hline$c_{\mathrm{M}, \mathrm{t}}^{*}$ & $\begin{array}{l}\text { Total concentration of metal in the entire } \\
\text { dispersion }\left(\mathrm{mol} \mathrm{m}^{-3}\right)\end{array}$ \\
\hline$c_{\mathrm{M}, \mathrm{f}}^{\mathrm{DL}}$ & $\begin{array}{l}\text { Local concentration of free metal ion in the } \\
\text { intraparticulate double layer }\left(\mathrm{mol} \mathrm{m}^{-3}\right)\end{array}$ \\
\hline$c_{\mathrm{M}, \mathrm{f}}^{\mathrm{D}}$ & $\begin{array}{l}\text { Local concentration of free metal ion in the } \\
\text { Donnan volume }\left(\mathrm{mol} \mathrm{m}^{-3}\right)\end{array}$ \\
\hline
\end{tabular}


$c_{\mathrm{M}, \mathrm{cond}}^{\mathrm{DL}} \quad$ Local concentration of condensed metal ion in the intraparticulate double layer $\left(\mathrm{mol} \mathrm{m}^{-3}\right)$

$c_{\mathrm{M}, \mathrm{f}} \quad$ Local average concentration of free metal ion in the particle body $\left(\varphi_{\mathrm{DL}} c_{\mathrm{M}, \mathrm{f}}^{\mathrm{DL}}+\varphi_{\mathrm{D}} c_{\mathrm{M}, \mathrm{f}}^{\mathrm{D}}\right)\left(\mathrm{mol} \mathrm{m}^{-3}\right)$

$c_{\mathrm{M}, \mathrm{t}}$ Local total concentration of all forms of $\mathrm{M}$ in the particle body $\left(\mathrm{mol} \mathrm{m}^{-3}\right)$

$c_{\mathrm{MS}} \quad$ Local concentration of inner-sphere complex within the particle volume $\left(\mathrm{mol} \mathrm{m}^{-3}\right)$

$c_{\mathrm{MHA}}^{*}$

$c_{\mathrm{MS}}^{*}$

$c_{\mathrm{S}, \mathrm{t}}^{*}$

$c_{\mathrm{S}}^{*}$

$c_{\mathrm{S}, \mathrm{t}}$

$c_{\mathrm{S}}$

$e$

$\bar{f}_{\mathrm{B}, \mathrm{M}}$

$\bar{f}_{\mathrm{C}}$

$f^{\mathrm{os}}$

K

$K^{\mathrm{os}}$

$\bar{K}_{\text {app }}$

$\bar{K}_{\text {int }}$

$\kappa^{-1}$

$\kappa_{\mathrm{p}}^{-1}$

$\ell_{\mathrm{DL}}$

$\ell_{\mathrm{C}}$

$n_{\mathrm{S}}$

$r_{\mathrm{p}}$

$V_{\mathrm{D}}$

$V_{\mathrm{DL}}$

$V^{\text {os }}$

$V_{\mathrm{p}}$

$\psi_{\mathrm{D}}$

$\psi_{\mathrm{DL}}$

$\bar{\rho}_{\mathrm{DL}}$

$\rho_{\mathrm{p}}$

$\varphi_{\mathrm{DL}}$

$\varphi_{\mathrm{D}}$
Smeared-out concentration of the total $\mathrm{M}$ associated with HA particles $\left(\mathrm{mol} \mathrm{m}^{-3}\right)$ Smeared-out concentration of inner-sphere bound $\mathrm{M}\left(\mathrm{mol} \mathrm{m}^{-3}\right)$

Smeared-out total concentration of reactive sites $\left(\mathrm{mol} \mathrm{m}^{-3}\right)$

Smeared-out concentration of reactive sites $\left(\mathrm{mol} \mathrm{m}{ }^{-3}\right)$

Local total concentration of reactive sites within the particle volume $\left(\mathrm{mol} \mathrm{m}^{-3}\right)$

Local concentration of reactive sites within the particle volume $\left(\mathrm{mol} \mathrm{m}^{-3}\right)$

Elementary charge

Donnan partitioning factor for the metal ion Condensation factor

Concentration enhancement factor for $\mathbf{M}^{z+}$ in the outer-sphere volume of an individual ion-pair in simple ligand case

Conventional stability constant for a simple ligand $\left(\mathrm{m}^{3} \mathrm{~mol}^{-1}\right)$

Outer-sphere ion pair stability constant $\left(\mathrm{m}^{3} \mathrm{~mol}^{-1}\right)$ Apparent average stability constant for MHA based on smeared-out concentrations $\left(\mathrm{m}^{3} \mathrm{~mol}^{-1}\right)$ Intrinsic average stability constant for innersphere MS based on local intraparticulate concentrations $\left(\mathrm{m}^{3} \mathrm{~mol}^{-1}\right)$

Debye length in the bulk aqueous medium (m)

Intraparticulate Debye length (m)

Thickness of the intraparticulate condensation zone $(\mathrm{m})$

Separation distance between charged sites (m)

Number of reactive sites per particle

Particle radius $(\mathrm{m})$

Volume of the Donnan phase $\left(\mathrm{m}^{3}\right)$

Volume of the intraparticulate double layer zone $\left(\mathrm{m}^{3}\right)$

Outer-sphere volume for an ion pair between $\mathrm{M}_{\mathrm{aq}}^{2+}$ and an individual site $\mathrm{S}\left(\mathrm{m}^{3}\right)$

Volume of the NP entity $\left(\mathrm{m}^{3}\right)$

Donnan potential (V)

Potential at a given spatial position within the intraparticulate double layer (V)

Average volume charge density in the double layer $\left(\mathrm{mol} e \mathrm{~m}^{-3}\right)$

Volume charge density in the particle body $\left(\mathrm{mol} e \mathrm{~m}^{-3}\right)$

Particle volume fraction of the double layer zone

Particle volume fraction of the Donnan zone
$\theta_{\mathbf{M}}$

Ratio of concentrations of inner-sphere complexes and reactive sites

\section{References}

1 H. P. van Leeuwen, J. Buffle, J. F. L. Duval and R. M. Town, Langmuir, 2013, 29, 10297-10302.

2 H. P. van Leeuwen and J. Buffle, Environ. Sci. Technol., 2009, 43, 7175-7183.

3 J. F. L. Duval, K. J. Wilkinson, H. P. van Leeuwen and J. Buffle, Environ. Sci. Technol., 2005, 39, 6435-6445.

$4 \mathrm{~J}$. Buffle, Complexation Reactions in Aquatic Systems: An Analytical Approach, Ellis Horwood, Chichester, UK, 1988.

5 B. Manunza, S. Deiana, V. Maddau, C. Gessa and R. Seeber, Soil Sci. Soc. Am. J., 1995, 59, 1570-1574.

6 R. D. Guy and C. L. Chakrabarti, Can. J. Chem., 1976, 54, 2600-2611.

7 L. K. Koopal, T. Saito, J. P. Pinheiro and W. H. van Riemsdijk, Colloids Surf., A, 2005, 265, 40-54.

8 E. Tipping, Aquat. Geochem., 1998, 4, 3-48.

9 P. Warwick, A. Hall, S. J. King, J. Zhu and J. van der Lee, Radiochim. Acta, 1998, 81, 215-221.

10 A. P. Robertson and J. O. Leckie, Environ. Sci. Technol., 1999, 33, 786-795.

11 R. Marsac, M. Davranche, G. Gruau, M. Bouhnik-Le Coz and A. Dia, Geochim. Cosmochim. Acta, 2011, 75, 5625-5637.

12 A. C. Montenegro, S. Orsetti and F. V. Molina, Environ. Chem., 2014, 11, 318-332.

13 N. D. Bryan, D. M. Jones, M. Appleton, F. R. Livens, M. N. Jones, P. Warwick, S. King and A. Hall, Phys. Chem. Chem. Phys., 2000, 2, 1291-1300.

14 M. F. Benedetti, W. H. van Riemsdijk and L. K. Koopal, Environ. Sci. Technol., 1996, 30, 1805-1813.

15 H. P. van Leeuwen and R. M. Town, Environ. Chem., 2016, 13, 76-83.

16 G. S. Manning, Acc. Chem. Res., 1979, 12, 443-449.

17 G. S. Manning, Biophys. Chem., 1977, 7, 95-102.

18 E. Garcia-Fernandez, P. M. R. Paulo and S. M. B. Costa, Phys. Chem. Chem. Phys., 2015, 17, 4319-4327.

19 M. Majtyka and J. Kłos, Phys. Chem. Chem. Phys., 2007, 9, 2284-2292.

20 Q. R. Huang, P. L. Dubin, C. N. Moorefield and G. R. Newkome, J. Phys. Chem. B, 2000, 104, 898-904.

21 Z. Li, A. K. van Dyk, S. J. Fitzwater, K. A. Fichthorn and S. T. Milner, Langmuir, 2016, 32, 428-441.

22 K. Krishnan and R. A. Plane, J. Am. Chem. Soc., 1968, 90, 3195-3200.

23 L. A. Clapp, C. J. Siddons, J. R. Whitehead, D. G. van Derveer, R. D. Rogers, S. T. Griffin, S. B. Jones and R. D. Hancock, Inorg. Chem., 2005, 44, 8495-8502.

24 M. Eigen, Pure Appl. Chem., 1963, 6, 97-115.

25 R. M. Fuoss, J. Am. Chem. Soc., 1958, 80, 5059-5061.

26 H. P. van Leeuwen, R. M. Town and J. Buffle, J. Phys. Chem. A, 2007, 111, 2115-2121. 
27 P. G. Daniele, A. De Robertis, C. De Stefano, S. Sammartano and C. Rigano, J. Chem. Soc., Dalton Trans., 1985, 2353-2361.

28 T. Miyajima, K. Yoshida, Y. Kanegae, H. Tohfuku and J. A. Marinsky, React. Polym., 1991, 15, 55-62.

29 R. F. M. J. Cleven, Heavy Metal/Polyacid Interaction. An Electrochemical Study of the Binding of $C d($ II), $P b($ II) and Zn(II) to Polycarboxylic Acids and Humic Acids, PhD thesis, Wageningen Agricultural University, 1984.

30 R. M. Town, J. Buffle, J. F. L. Duval and H. P. van Leeuwen, J. Phys. Chem. A, 2013, 117, 7643-7654.

31 H. Ohshima and T. Kondo, Biophys. Chem., 1990, 38, 117-122.

32 L. P. Yezek and H. P. van Leeuwen, Langmuir, 2005, 21, 10342-10347.

33 J. F. L. Duval, Langmuir, 2005, 21, 3247-3258.

34 R. Vermeer, Interactions Between Humic Acid and Hematite and Their Effects on Metal Ion Speciation, PhD thesis, Wageningen Agricultural University, 1996.

35 W. F. Tan, L. K. Koopal and W. Norde, Environ. Sci. Technol., 2009, 43, 591-596.

36 J. P. Pinheiro, A. M. Mota, J. M. R. d'Oliveira and J. M. G. Martinho, Anal. Chim. Acta, 1996, 329, 15-24.

37 M. Hosse and K. J. Wilkinson, Environ. Sci. Technol., 2001, 35, 4301-4306.

38 J. Puy, J. Galceran, C. Huidobro, E. Companys, N. Samper, J. L. Garcés and F. Mas, Environ. Sci. Technol., 2008, 42, 9289-9295.

39 G. Abate and J. C. Masini, Org. Geochem., 2002, 33, 1171-1182.

40 H. M. V. M. Soares, P. C. F. L. Conde, A. A. N. Almeida and M. T. S. D. Vasconcelos, Anal. Chim. Acta, 1999, 394, 325-335.

41 H. E. Mash, Y.-P. Chen, L. Sigg, R. Hari and H.-B. Xue, Anal. Chem., 2003, 75, 671-677.

42 R. M. Town and H. P. van Leeuwen, Electroanalysis, 2004, 16, 458-471.

43 J. Galceran, E. Companys, J. Puy, J. Cecília and J. L. Garcés, J. Electroanal. Chem., 2004, 566, 95-109.
44 R. M. Town and H. P. van Leeuwen, J. Electroanal. Chem., 2001, 509, 58-65.

45 M. A. G. T. van den Hoop, H. P. van Leeuwen and R. F. M. J. Cleven, Anal. Chim. Acta, 1990, 232, 141-148.

46 A. van der Wal, M. Minor, W. Norde, A. J. B. Zehnder and J. Lyklema, Langmuir, 1997, 13, 165-171.

47 H. Ohshima and S. Ohki, Biophys. J., 1985, 47, 673-678.

48 H. P. van Leeuwen, R. F. M. J. Cleven and P. Valenta, Pure Appl. Chem., 1991, 63, 1251-1268.

49 I. Christl, Environ. Chem., 2012, 9, 89-96.

50 J. G. Hering and F. M. M. Morel, Environ. Sci. Technol., 1988, 22, 1234-1237.

51 R. von Wandruszka, Soil Sci., 1998, 163, 921-930.

52 I. Christl, A. Metzger, I. Heidmann and R. Kretzschmar, Environ. Sci. Technol., 2005, 39, 5319-5326.

53 A. Liu and R. D. Gonzalez, Langmuir, 2000, 16, 3902-3909.

54 E. Companys, J. Puy and J. Galceran, Environ. Chem., 2007, 4, 347-354.

55 A. W. P. Vermeer, J. K. McCulloch, W. H. van Riemsdijk and L. K. Koopal, Environ. Sci. Technol., 1999, 33, 3892-3897.

56 C. Kolokassidou and I. Pashalidis, Radiochim. Acta, 2006, 94, 549-552.

57 J. Xiong, L. K. Koopal, W. F. Tan, L. C. Fang, M. X. Wang, W. Zhao, F. Liu, J. Zhang and L. P. Weng, Environ. Sci. Technol., 2013, 47, 11634-11642.

58 I. Christl, C. J. Milne, D. G. Kinniburgh and R. Kretzschmar, Environ. Sci. Technol., 2001, 35, 2512-2517.

59 Y. Marcus, Chem. Rev., 1988, 88, 1475-1498.

60 H. P. van Leeuwen, J. Buffle and R. M. Town, Langmuir, 2012, 28, 227-234.

61 R. M. Town and H. P. van Leeuwen, Environ. Chem., 2014, 11, 196-205.

62 H. P. van Leeuwen, J. Radioanal. Nucl. Chem., 2000, 246, 487-492.

63 P. D. Polyakov and J. F. L. Duval, Phys. Chem. Chem. Phys., 2014, 16, 1999-2010.

64 S. Jansen, R. Blust and H. P. van Leeuwen, Environ. Sci. Technol., 2002, 36, 2164-2170. 